DOI 10.19195/2084-2546.25.10

\author{
ANNA WENDORFF \\ Uniwersytet Łódzki \\ Correo: anna.wendorff@uni.lodz.pl
}

\title{
Narrar la experiencia de la emigración. Los polacos en Misiones (Argentina)
}

\author{
Palabras clave: emigración polaca - Misiones — identidad local — identidad \\ global - narración de identidades.
}

El presente artículo es un estudio especialmente dedicado al análisis de los métodos de investigación aplicados a las experiencias de inmigración. No obstante, queremos aclarar que no se expondrán ejemplos de los casos de estudio, sino que únicamente se hace referencia a la dimensión científica para abordar el tema.

Misiones es una región que históricamente ha emergido sobre la base de continuos "desplazamientos": étnicos, sociales, culturales, políticos, etc. Los primeros y los que más han sufrido estas transformaciones han sido por supuesto las comunidades originarias del lugar. Inicialmente debido a las influencias que marcaron los grupos colonizadores y posteriormente por otros sectores.

Fueron los misioneros jesuíticos, junto con sus proyectos de transformación, los que trataron de dar al "autóctono" del lugar una dimensión que ellos consideraron justa y apropiada. Pero, así como intentaron producir cambios culturales en los diferentes grupos étnicos de la región, los jesuitas también encontraron resistencia por parte de los suyos. Estas formas de resistencia y de desmembración del tejido social se instalaron posteriormente en grupos de poder, clases, élites, etc. Cada vez más, familias con cierto tipo de poder (fundamentalmente político y económico) se fueron adueñando, aunque bastante paulatinamente, de considerables porciones de tierra y también de culturas

* Es becaria de la Fundación de la Universidad de Łódź para el año 2017.

** Investigación realizada durante la estancia postdoctoral desde el 15 de diciembre de 2014 hasta el 15 de junio de 2015 en el Departamento de Antropología Social en la Universidad Nacional de Misiones en Posadas (Argentina) en el marco del grant EURICA, Erasmus Mundus Action 2, número 2013-2587, strand 1, lot 15 . 
en el vasto y complejo sistema ecológico que abunda en Misiones. No obstante, ello no implica que la devastación sistemática, implementada desde la colonia hasta casi nuestros días, haya logrado sus objetivos; entre otros el de modificar la estructura de los diversos modelos culturales que cohabitan en la región. A partir pues de estas "pugnas", hacen presencia una gran cantidad de mecanismos y formas de resistencia (en ocasiones solapadas con diferentes prácticas sociales comunes) que llevan a los pueblos y a sus diferentes grupos étnicos a encontrar formas de sobrevivencia frente a estas "razzias culturales", "razzias históricas", "razzias lingüísticas", etc. Es necesario aclarar que los mecanismos de resistencia interpuestos para romper los ejes de dominación no se imponen como proyectos programáticos únicos. Son más bien parte de ciertas estructuras que funcionan y actúan como "mecanismos de defensa", y que emergen desde los diversos grupos societales que cohabitan en la zona. Así, las distintas prácticas de resistencia se expresan en concordancia con la formación de su "temperamento".

Estos mismos mecanismos de presión y lucha dados entre los diferentes grupos que operaron en la Misiones originaria, se aplicaron de la misma manera a un nutrido grupo de polacos que emigró a Argentina a finales del siglo XIX. Y este mismo doble sentido, que emerge precisamente de aquel que está aventurando vida en tierra extranjera, es decir, el colonizador-colonizado, devino en portavoz de la mostración y configuración de una complejidad identitaria que se articulaba a la vez sobre un mapa (podemos decir) aún más complejo. Así fue la historia de los polacos en Misiones, que se igualaron a las desplazadas etnias guaraníes.

Las culturas que con el paso de la historia se han visto marginadas, también han podido ver cómo se diezma a su propia y particular condición histórica. Este es el resultado que ha operado, a la vez en los pueblos originarios y a la vez en los inmigrantes.

Derivado de este proceso, podemos afirmar que Misiones se transformó pues en una región de inmigrantes. Esta profusa inmigración trajo como resultado que se gestaran importantes "intercambios simbólicos"1 entre las diversas comunidades de cohabitación. Los intercambios simbólicos quieren afirmar la idea de que entre un actor social y otro puede haber no solo un proceso de "diálogo" y expresión de diferencias en ese diálogo, sino de afirmación y concurrencia de similitudes en las ideas, en la vida, en las formas de ver el mundo, etc.

Para que ambas comunidades misioneras y polacas pudieran entrar en "profundo" contacto, esos intercambios deberían definirse a partir de un marco de "acomodos" y "re-acomodos" sociales que se produjeran de acuerdo con los intereses particulares de cada uno.

Para que podamos tener una comprensión más definida debemos decir que confluyen en la zona brasileños, paraguayos, argentinos; miembros de

${ }^{1}$ J. Baudrillard, El intercambio simbólico y la muerte, Caracas, Monte Ávila, 1980. 
tres comunidades que a pesar de su cercanía geográfica están conectados bajo un tipo de relaciones que borra temporalmente, o casi permanentemente, la frontera territorial. La llegada de las comunidades de polacos agrega un cuarto grupo, bastante ajeno al resto y que marca una diferencia sustantiva con respecto a la totalidad. Sin embargo, termina siendo una comunidad más que se suma a este complejo entramado social.

Desde finales del siglo XIX (1897) los migrantes-inmigrantes han visto cómo paulatinamente comunidades de otras latitudes, en extremo diferentes (en este caso los polacos) se han adentrado en una tierra que puede significar o que al menos significó para estos nuevos colonos un raro y exótico paisaje, y en la cual de pronto sintieron (como todo extranjero) la necesidad de estar y conectar sus raíces con la de estos pueblos. Esta dicotomía nos llevó a plantearnos un primer punto de partida para analizar adecuadamente las confluencias de relaciones establecidas: ¿Por qué este "sujeto" (polaco) se acomoda en una cierta región que está tan alejada, entendida en todos los modos, de su propio origen? La segunda pregunta que puede derivar de aquí es: ¿Qué contenidos son emergentes y cómo colarlos con los de la localidad? Dicho de otro modo, es lo que definiríamos como: "la palabra que conecta...", "el modo que conecta..."; es decir, de qué manera los polacos que se instalaron en Misiones encontraron un puente de relaciones entre ellos y los otros, como para dar cuenta de su "existencia" y así mismo de la "existencia del otro".

Es evidente que son temáticas y conflictos presentes en diferentes contextos y expresados bajo diferentes modalidades, por ejemplo tal como se describe en la solapa del libro Wanda, mi pueblo ${ }^{2}$ :

La colectividad polaca de Wanda rinde su homenaje a la tierra lejana y a este suelo argentino que la cobijó, uniendo en poemas el sentimiento de su gente. Este puñado de trabajos literarios, es el resultado del primer concurso de poesías ilustradas organizado por nuestra institución, con motivo del $69^{\circ}$ aniversario de Colonia Wanda ${ }^{3}$.

Para tener una idea más adecuada de las relaciones sociales creadas entre las diferentes etnias y el grupo de los inmigrantes, la investigadora Claudia Stefanetti Kojrowicz en su artículo "Los inmigrantes polacos en Misiones y su primer pan de maíz"4 nos reporta cómo y en qué cantidad se dio la llegada del primer grupo de polacos a tierra misionera. Según Stefanetti Kojrowicz los primeros polacos que llegan a Argentina (69 de acuerdo con su información) lo hacen porque a pesar de que su destino primero era EE.UU., no les permiten ingresar al país en cuestión y son desviados a Argentina. Tal como nos lo dice Stefanetti Kojrowicz:

${ }^{2}$ M.A. Wall et al., Wanda, mi pueblo, Wanda (Misiones-Argentina), Ediciones de Wanda, 2005.

${ }^{3}$ Ibidem. La inscripción aparece en la solapa del libro.

${ }^{4}$ C. Stefanetti Kojrowicz, "Los inmigrantes polacos en Misiones y su primer pan de maíz", en: El águila blanca, 2002, pp. 1-16, <http://www.elaguilablanca.com.ar/Descargas/Primerpandemaiz. pdf $>, 4$ de mayo de 2017. 
Si recordamos que provenían de un imperio multinacional es fácil imaginar que al ignorar la idea de Estado nacional, tal como ya se la entendía en estas latitudes, les haya parecido que sería lo mismo ir a los Estados Unidos o la Argentina, pues todo era América ${ }^{5}$.

La importancia de estos acontecimientos radica en que el fenómeno principal de nuestra investigación se fundaría en un número diverso de perspectivas y algunos conceptos. Debemos explicar primeramente que la fuente de los contenidos a analizar concurre por diferentes vías. Obviamente, las vías teóricas y las prácticas que se consustancian unas con otras. Las teóricas tienen fuentes en algunas tesis de Paul Ricoeur, Marie-Françoise Chanfrault-Duchet, Mijaíl Bajtín y en el tema de la metodología de los trabajos de Rosana Guber y más lejano en algunas categorizaciones de Jean Baudrillard. Las fuentes prácticas tienen sus representaciones y estructuras básicas por supuesto en los colonos polacos que han desarrollado una fuerte comunidad dentro de la región, la cual cuenta con encuentros, asociaciones, grupos de contacto, escuelas, etc. Hay una preeminencia fundamental por mantener el sentido de pertenencia no solo geográfico, sino como comunidad, y otro por sostenerlo y expresarlo como sujetos de una comunidad (nueva).

Así, si nos remitimos únicamente al ámbito teórico, los conceptos fundamentales que manejaremos para la intervención de nuestro análisis son:

1. El concepto de "identidad narrativa" de Paul Ricoeur. Ricoeur ha establecido en su libro Tiempo y Narración ${ }^{6}$ las diferencias y las cercanías dadas entre relato histórico y el de ficción. A partir de esa idea podemos afirmar que, producto del complejo sistema de relaciones discursivas entre relato histórico y relato de ficción, se generan estas complejas identidades narrativas. Tal como lo propone Ricoeur, el relato no solo es el descriptor de un acontecimiento, sino el encadenamiento de un grueso de definiciones que elaboran a partir de metaficciones y/o autoficciones; pases, transferencias, encadenamientos, que "tejen" nuevas prácticas discursivas, prácticas de sentido y representaciones sociales.

a. Los polacos en Misiones construyen un micromundo revelado en estas discursividades.

b. Así como ha habido un desplazamiento fronterizo, igualmente podemos encontrar desplazamientos discursivos.

2. La noción de historia oral, aquella en donde la oralidad no es solo un denominador común del habla, sino también una forma profunda de expresión y de la historia de ese "habla". Así, la historia oral se define como relato (básicamente, esencial, simple de la vida cotidiana) y también como habla histórica a la vez que construye un entramado más complejo. De acuerdo con esta idea, la propuesta de Daniel Brauer parece ajustarse a nuestra tesis y así esclarecer algunos de los planteamientos que se han ido presentando; entendi-

5 Ibidem, p. 7.

${ }^{6}$ P. Ricoeur, Tiempo y narración, vol. 1. Configuración del tiempo en el relato histórico, México, D.F., Siglo Veintiuno Editores, 2004. 
dos siempre en relación con los diferentes grupos que conviven en la región. Brauer expresa:

La tesis central de la monumental obra de Ricoeur, Tiempo y relato, es que "el tiempo deviene tiempo humano en la medida en que es articulado de manera narrativa; a la inversa, el relato es significativo en la medida en que dibuja los trazos de la experiencia temporal". Aquí el tiempo, y particularmente el pasado humano, no son reducidos al relato mismo, pero la experiencia narrativa es vista como constituyendo nuestro único e indirecto modo de acceso al tiempo histórico ${ }^{7}$.

3. Narrativas, discursos y oralidad: A partir de aquí se intentó desarrollar la idea de que los actores sociales de continuo se plantean y construyen un sinnúmero de narrativas de identidad. No obstante, en el mismo sentido también, que las identidades, identificaciones, construyen un cuerpo diverso de narrativas y discursos. De modo tal que, a través de sí, manifiestan y definen el qué somos, cómo somos, qué aspiraciones se tienen, etc. A partir de este doble (o quizá múltiple) rasero y de acuerdo con lo que nos propone MarieFrançoise Chanfrault-Duchet podemos entonces asumir que los "discursos" en los colonos manifestarán sus deseos, ideales, afirmaciones, y también sus esperanzas, impresiones, etc. Veamos lo que afirma Chanfrault-Duchet:

Since some confusion exists in oral history among the different products of an interview, it is important at the outset to define the life story accurately as both an object and a genre. Drawing on my past work, I define the life story through two features: (1) the specifically narrative and "literary" nature of the object produced in a dialogue; and (2) the social nature of the self dramatized in the narrative. The narrative dimension refers to the fact that the life story aims, by means of a coherent and global process, to account for the whole of the informant's life experience until the moment of the interview. This means that the narrative encompasses not only the temporal and causal organization of facts and events considered significant, but also the value judgments that make sense of this particular life experience. ${ }^{8}$

4. Revisión de los papeles sociales: Aquí está presente la multiplicidad de cambios y de transformaciones que surgen a partir de los "papeles"/"roles" que los actores sociales asumen. De la misma forma que en la literatura, en el campo mismo de la narración (y que opera aquel que entrevista) se determinan cambios de posición y perspectiva de acuerdo con los roles que se van asumiendo. Lo que querríamos hacer aquí se referiría a mirar la intención que ha construido el hablante, asumiendo un rol que puede estar significado en un texto escrito o en el relato propiamente dicho.

5. Polifonía Territorial (de acuerdo con lo que plantea Mijaíl Bajtín, a través de la categoría polifonía, en su libro Problemas de la poética de

${ }^{7}$ D. Brauer, "La fragilidad del pasado", en: M. Cruz (comp.), Hacia dónde va el pasado, Barcelona, Paidós, 2002, p. 44.

${ }^{8}$ M.F. Chanfrault-Duchet, "Narrative Structures, Social Models, and Symbolic Representation in the Life Story", en: S. Berger Gluck, D. Patai (eds.), Women's Words: The Feminist Practice of Oral History, New York, Routledge, 1991, p. 77. 
Dostoievski ${ }^{9}$ ): Se refiere no solamente a la conjunción de diversos grupos étnicos y culturales que conviven en una misma región, sino que esa diversidad (expresada fundamentalmente a través de las diferencias lingüísticas) lleva acorde así no una sola voz, sino múltiples voces. Un por doquier de voces que se manifiestan en diferentes planos: sociales, culturales, locales, geográficos y conviven entre sí y simultáneamente. En consecuencia, los que en 1897 recién llegan de Polonia, luego de una larga travesía, no decantan en Misiones su cultura "simple"; sino que fundan una nueva red, un nuevo mundo plagado de un complejo sistema de relaciones. No es efectivamente la sustitución de un esquema por otro (La información apunta a mostrar cómo se establece la polifonía territorial).

\section{Relato de vida, enfoque biográfico (Camino a una descripción narrativa para la construcción de una historia)}

Para poder abordar estas teorías, conceptos, y así mismo observar cómo se pueden colocar en las narrativas identitarias, se decidió que la metodología de trabajo a emplear debía profundizar en dos elementos principales, y que son a nuestro juicio formas concretas de relaciones con el tema. Nos referimos a los siguientes:

1. El relato de vida / el enfoque biográfico.

2. La entrevista como recolector no solo de información, sino también el proceso de hilación de gaffes, de acuerdo con lo que nos explica Rosana Guber en su libro La etnografía: método, campo y reflexividad ${ }^{10}$. Citemos:

[e]xplica, por un lado, la necesidad de los etnógrafos de basar su discurso —oral, escrito, teórico y empírico- en una instancia empírica específica repleta de rupturas y tropiezos, gaffes y contratiempos, lo que los antropólogos han bautizado "incidentes reveladores". Por otro lado, explica que "en la investigación de campo se constate que la vida imita a la teoría, porque el investigador entrenado en los aspectos más extraños hasta los más corrientes de la conducta humana, encuentra en su experiencia un ejemplo vivo de la literatura teórica a partir de la cual se formó"11.

Tal como se puede observar, esto nos conduce directamente a entender que los instrumentos de recolección de datos e información en el campo de investigación no enmarcan a los contenidos en un solo bloque, sino que los distribuyen más bien dispersándolos puesto que "el investigador social solo

\footnotetext{
${ }^{9}$ M.M. Bajtín, Problemas de la poética de Dostoievski, México, D.F., Fondo de Cultura Económica, 1986.

${ }^{10}$ R. Guber, La etnografia: método, campo y reflexividad, Bogotá, Grupo Editorial Norma, 2001.

${ }^{11}$ Ibidem, p. 18.
} 
puede conocer otros mundos a través de su propia exposición a ellos" ${ }^{\prime 2}$. El investigador social implementa un sistema que le permite reconocer, esta idea que Guber expresa como: "exposición a los mundos", y que según la misma teórica, se fundamente en que no hay instrumentos socioculturales de investigación que sean suficientemente plausibles para desmontar la totalidad de los análisis que pueda haber.

Guber, además, nos explica que durante el proceso de investigación, "la vida imita a la teoría", y a partir de allí nos permitimos afirmar previamente que ello generalmente no ocurre así. Los polacos entrevistados en Misiones revelan a través de sus discursos, ideas y expresiones, que en el contexto que se desplaza entre ficción e historia hay varios descriptores (elementos de significativa importancia) que exponen (ante la mirada del otro) diferentes esquemas para constituirse como tales. El modelo nos lleva a acercarnos directamente con el participante, "dejándolo que se desenvuelva" lo más libremente que pueda y, una vez allí, tratar de llegar a comprender las posturas conceptuales y los contenidos más profundos de su habla. Esto se puede ver en lo que nos afirma Stefanetti Kojrowicz:

La mayoría de los padres veían el aprendizaje escolar como un mal impuesto por el gobierno que alejaba a los niños de las ocupaciones verdaderamente útiles de la chacra. Se resignaron a enviarlos a la escuela sólo por temor a la ley que los obligaba. Al mismo tiempo que las autoridades nacionales trataban de "argentinizar" a los niños, el clero de origen polaco intentaba "polonizarlos" a través de la educación religiosa en polaco ${ }^{13}$.

También Stemplowski hace referencia (y de forma mucho más detallada) a la necesidad de Polonia de mantener sus costumbres, formas y lenguas en los que han emigrado, elemento que complicaría el sistema de relaciones entre los originarios del lugar (misioneros) y los "recién llegados" (polacos). En este sentido veamos que estos planteamientos se apoyan en las investigaciones que realizó Ryszard Stemplowski y de las cuales obtenemos algunas referencias a partir de su artículo "Los colonos eslavos del Nordeste Argentino (1897-1938). Problemática, fuentes e investigaciones en Polonia" ${ }^{14}$. Stemplowski afirma que:

Las escuelitas habían sido financiadas por inmigrantes (las subvenciones argentinas ocurrían rara vez). Sólo en los años 1928-1932 el gobierno polaco trataba de establecer una red de las escuelas polacas para los eslavos en Misiones, enviando maestros, dinero e ideas. Había una tentativa para desarrollar la conciencia nacional polaca entre los colonos eslavos, ya que una pérdida de la identidad nacional polaca entre los emigrantes de Polonia, cualesquiera que sea el país donde se establecieron, se la percibía en Polonia como el debilitamiento de la raison d'Etat polaca. Este proyecto oficial polaco nunca había sido planteado de modo realista y no tuvo éxito. A los fines de los años treinta, la enseñanza polaca no constituyó más de unos

12 Ibidem, p. 17.

13 C. Stefanetti Kojrowicz, op. cit., p. 9.

14 R. Stemplowski, "Los colonos eslavos del Nordeste Argentino (1897-1938). Problemática, fuentes e investigaciones en Polonia", Estudios Latinoamericanos, 10, 1985, pp. 169-183. 
pocos, breves y poco efectivos cursos de idioma polaco, con la participación muy reducida. Las llamadas escuelas polacas nunca jugaban, entre los inmigrantes polacos, un papel de la institución principal de la educación: tal papel lo desempeñaron las escuelas argentinas de Misiones. El proceso de la argentinización fue muy rápido ${ }^{15}$.

Parece pues bastante claro que este universo de tensiones afectó la relación entre los misioneros y las colonias eslavas recién llegadas a Misiones. No obstante, no solo ello ha sido el conjunto de los cambios dados a partir de la relación entre los inmigrantes y los de origen. Aquellas narrativas de identidad con las que los inmigrantes arriban, se ven prontamente ajustadas a un cuerpo de mecanismos discursivos ficcionales, metaficcionales, autoficcionales nuevos. Estos nuevos procedimientos discursivos puestos tanto en el habla como en la escritura, nos permite comprender de qué forma se produce la propia transformación y adecuación que los diversos grupos sociales adquieren a través del tiempo. En consecuencia, terminan adaptándose al nuevo "ecosistema" en el que viven y se desenvuelven. De modo que no solo producen cambios en sus hablas, sus escrituras, sus relatos, sino que también en el marco de sus hábitos de vida, de sus ideas, de sus pensamientos y de sus memorias. La inmigración siempre es una nueva forma de narración del pasado, pero también del presente de vida.

\section{Referencias bibliográficas}

\section{BAJTÍN M.M.}

1986 Problemas de la poética de Dostoievski, México, D.F., Fondo de Cultura Económica. BAUDRILLARD J.

1980 El intercambio simbólico y la muerte, Caracas, Monte Ávila. BRAUER D.

2002 "La fragilidad del pasado", en: Cruz M. (comp.), Hacia dónde va el pasado, Barcelona, Paidós, pp. 35-52.

CHANFRAULT-DUCHET M.F.

1991 "Narrative Structures, Social Models, and Symbolic Representation in the Life Story", en: Berger Gluck S., Patai D. (eds.), Women's Words: The Feminist Practice of Oral History, New York, Routledge, pp. 77-92.

GUBER R.

2001 La etnografía: método, campo y reflexividad, Bogotá, Grupo Editorial Norma. RICOEUR P.

2004 Tiempo y narración, vol. 1. Configuración del tiempo en el relato histórico, México, D.F., Siglo Veintiuno Editores.

STEFANETTI KOJROWICZ C.

2002 "Los inmigrantes polacos en Misiones y su primer pan de maíz", en: El águila blanca, 2002, pp. 1-16, <http://www.elaguilablanca.com.ar/Descargas/Primerpandemaiz.pdf`, 4 de mayo de 2017.

${ }^{15}$ Ibidem, p. 183. 
STEMPLOWSKI R.

1985 "Los colonos eslavos del Nordeste Argentino (1897-1938). Problemática, fuentes e investigaciones en Polonia", Estudios Latinoamericanos, 10, pp. 169-183.

WALL M.A. et al.

2005 Wanda, mi pueblo, Wanda (Misiones-Argentina), Ediciones de Wanda.

\section{Narrating the experience of emigration: Poles in Misiones (Argentina)} narration.

Keywords: Polish emigration - Misiones — local identity — global identity — identity

\section{Abstract}

The object of this study is to analyze how life accounts, histories, and testimonials of the Polish immigration in Misiones, Argentina, are constructed. The methodology employed for the analysis consists of: the ethnographic method, life accounts, biographic perspectives and research interviews, not only for information concerning the manner, but also the process of putting together gaffes, according to what Rosana Guber explains in her book La etnografia: método, campo $y$ reflexividad. The corpus of data was formed by Polish residents of the province of Misiones, Argentina. The results of the investigation determined that: their discourse acts as narration and not only as personal stories, constructs new identifying models and still others that refer to their original sources. As a corollary, the "new" identities have their manners of expression which are manifested via a series of discursive forms that characterize the speaker, such as the use of narrations that form an integral part of them. Finally, the conclusion suggests that the presence of the Polish immigrant in the province of Misiones affirms the concept of territorial polyphony and of narrative identities, since we can assume that in the narrative constructions similarly we will find discursive and metadiscursive constructions that propose identifying and historic relationships.

Fecha de recepción: 28.05.2017

Fecha de aceptación: 4.12.2017 